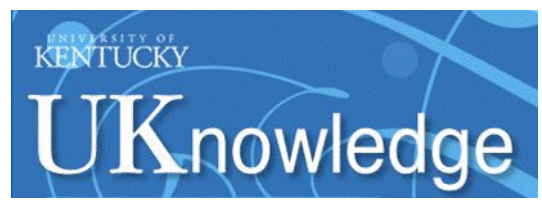

University of Kentucky

UKnowledge

\title{
$5-2019$
}

\section{Predicting Habitat Choice after Rapid Environmental Change}

Philip H. Crowley

University of Kentucky, pcrowley@email.uky.edu

Pete C. Trimmer

University of California, Davis

Orr Spiegel

Tel Aviv University, Israel

Sean M. Ehlman

University of California, Davis

William S. Cuello

University of California, Davis

See next page for additional authors

Follow this and additional works at: https://uknowledge.uky.edu/biology_facpub

Part of the Biology Commons, Environmental Sciences Commons, and the Zoology Commons

Right click to open a feedback form in a new tab to let us know how this document benefits you.

\section{Repository Citation}

Crowley, Philip H.; Trimmer, Pete C.; Spiegel, Orr; Ehlman, Sean M.; Cuello, William S.; and Sih, Andrew, "Predicting Habitat Choice after Rapid Environmental Change" (2019). Biology Faculty Publications. 193. https://uknowledge.uky.edu/biology_facpub/193

This Article is brought to you for free and open access by the Biology at UKnowledge. It has been accepted for inclusion in Biology Faculty Publications by an authorized administrator of UKnowledge. For more information, please contact UKnowledge@lsv.uky.edu. 


\section{Predicting Habitat Choice after Rapid Environmental Change}

Digital Object Identifier (DOI)

https://doi.org/10.1086/702590

\section{Notes/Citation Information}

Published in The American Naturalist, v. 193, no. 5.

(c) 2019 by The University of Chicago. All rights reserved.

The copyright holder has granted the permission for posting the article here.

Authors

Philip H. Crowley, Pete C. Trimmer, Orr Spiegel, Sean M. Ehlman, William S. Cuello, and Andrew Sih 


\title{
Predicting Habitat Choice after Rapid Environmental Change
}

\author{
Philip H. Crowley, ${ }^{1}$ Pete C. Trimmer, ${ }^{2,3, \star}$ Orr Spiegel, ${ }^{4}$ Sean M. Ehlman, ${ }^{2}$ William S. Cuello, ${ }^{2}$ \\ and Andrew $\mathrm{Sih}^{2}$
}

1. Department of Biology, University of Kentucky, Lexington, Kentucky 40506; 2. Department of Environmental Science and Policy, University of California, Davis, California 95616; 3. Evolutionsbiologie, Universität Bielefeld, Konsequenz 45, 33615 Bielefeld, Germany; 4. School of Zoology, Faculty of Life Sciences, Tel Aviv University, Tel Aviv 69978, Israel

Submitted November 15, 2017; Accepted November 13, 2018; Electronically published March 13, 2019

Online enhancements: appendixes, supplemental material.

Aвstract: Decisions made while searching for settlement sites (e.g. nesting, oviposition) often have major fitness implications. Despite numerous case studies, we lack theory to explain why some species are thriving while others are making poor habitat choices after environmental change. We develop a model to predict (1) which kinds of environmental change have larger, negative effects on fitness, (2) how evolutionary history affects susceptibility to environmental change, and (3) how much lost fitness can be recovered via readjustment after environmental change. We model the common scenario where animals search an otherwise inhospitable matrix, encountering habitats of varying quality and settling when finding a habitat better than a threshold quality level. We consider decisions and fitness before environmental change, immediately following change (assuming that animals continue to use their previously adaptive decision rules), and after optimal readjustment (e.g., via learning or evolution). We find that decreases in survival per time step searching and declines in habitat quality or availability generally have stronger negative effects than reduced season duration. Animals that were adapted to good conditions remained choosy after conditions declined and thus suffered more from environmental change than those adapted to poor conditions. Readjustment recovered much of the fitness lost through a reduction in average habitat quality but recovered much less following reductions in habitat availability or survival while searching. Our model offers novel predictions for empiricists to test as well as suggestions for prioritizing alternative mitigation steps.

Keywords: adaptive behavior, habitat loss, human-induced rapid environmental change (HIREC), search costs, habitat selection, natal dispersal.

\section{Introduction}

Human-induced rapid environmental change (HIREC; Sih et al. 2011) is having substantial and often negative effects on ecological systems around the world (Sih et al. 2011; Candolin and Wong 2012; Sih 2013; Wong and Candolin

\footnotetext{
* Corresponding author; email: pete.trimmer@gmail.com.
}

Am. Nat. 2019. Vol. 193, pp. 619-632. @ 2019 by The University of Chicago. 0003-0147/2019/19305-58078\$15.00. All rights reserved.

DOI: $10.1086 / 702590$
2015). One major aspect of HIREC is habitat change driven by both global and local changes, such as habitat loss, fragmentation, shifts in habitat quality in remaining patches, and altered costs of movement in the matrix between patches (Sih et al. 2000; Fahrig 2003; Burns and Grear 2008). Thus, understanding how animals choose their habitat under these abruptly altered conditions is an important goal. Some species appear to have adjusted well to habitat change (e.g., invasive species and urban exploiters; Kark et al. 2007), while others are making faulty decisions through inability to adjust or by responding to misleading cues, leading to ecological traps (Robertson et al. 2013; Hale and Swearer 2016). The broad topic we explore is how behavioral characteristics (shaped by prior environments) affect the impact of environmental changes on species' fitness (usually negatively, but not always) as well as species' potential to readapt after such changes. While we have numerous case studies, there is surprisingly little theory generating predictions on which species should adjust well and which might be more vulnerable to environmental changes as a result of poor habitat selection decisions. Ideally, such theory should expand previous models (Sih et al. 2011, 2016) to generate novel and testable predictions for explaining the relative success of different species in adjusting to habitat change and which aspects of habitat change are more problematic.

As a first step to developing a tractable model approach to address how environmental change might influence animals through their behavioral choices, we focus on situations where animals make a single habitat choice, which may have a large effect on fitness (Manly et al. 2007). While some realworld scenarios involve numerous repeated habitat choices (e.g., when foraging), the situation that we model (e.g., choice of a new territory or breeding site, such as a nesting site for birds or an oviposition site for insects or amphibians) is common and often critically important for fitness. For illustration, we use natal dispersal-relocation from a birthplace to a future home or breeding location - as the focal example throughout the text, but note that similar logic can be applied 
broadly across parallel types of site selection in which site choice has large impacts on the fitness of individuals.

In brief, we examine how well decision rules for habitat choice that were adaptive in local conditions before habitat change perform after habitat change and show how differences in evolutionary history may result in different sensitivities to environmental change. Unlike gradual environmental changes that allow for adaptation (i.e., adaptive tracking), rapid environmental changes often produce a mismatch between evolutionary history and current conditions. Such changes can be caused by natural disturbances (volcanic eruptions, massive wildfires, hurricanes, etc.) but are commonly driven by anthropogenic pressures that may alter the environment at unprecedented rates (Barnosky et al. 2011; Burrows et al. 2011). To investigate this topic, we extended a natal-dispersal model by Stamps et al. (2005) that is a variant of optimal-search models that have been used for various important decisions (e.g., diet, patch, or mate choice; see app. A; apps. A-C are available online). We assume that before the change, the species has adapted to its typical environment with decision rules that are appropriate for those conditions. We further assume that after rapid environmental change, the animal does not adjust its decision rule immediately. Many animals might not adjust quickly because as they begin searching, they lack good information on the change in habitat availability, average habitat quality, mortality risk while searching, or season length.

Depending on the extent of evolutionary mismatch between past and present conditions, those previously adaptive criteria might work well despite the change or might result in poor, or even egregiously maladaptive, decisions. Generations later, if the species has survived the severe conditions imposed by the rapid environmental change, readaptation is predicted to bring traits back in line with the environment. This basic approach is summarized in figure 1 .

We apply this modeling approach to generate predictions on how variation in settlement rules creates variation in species' responses to environmental changes, both immediately after habitat deterioration and generations later, fol-

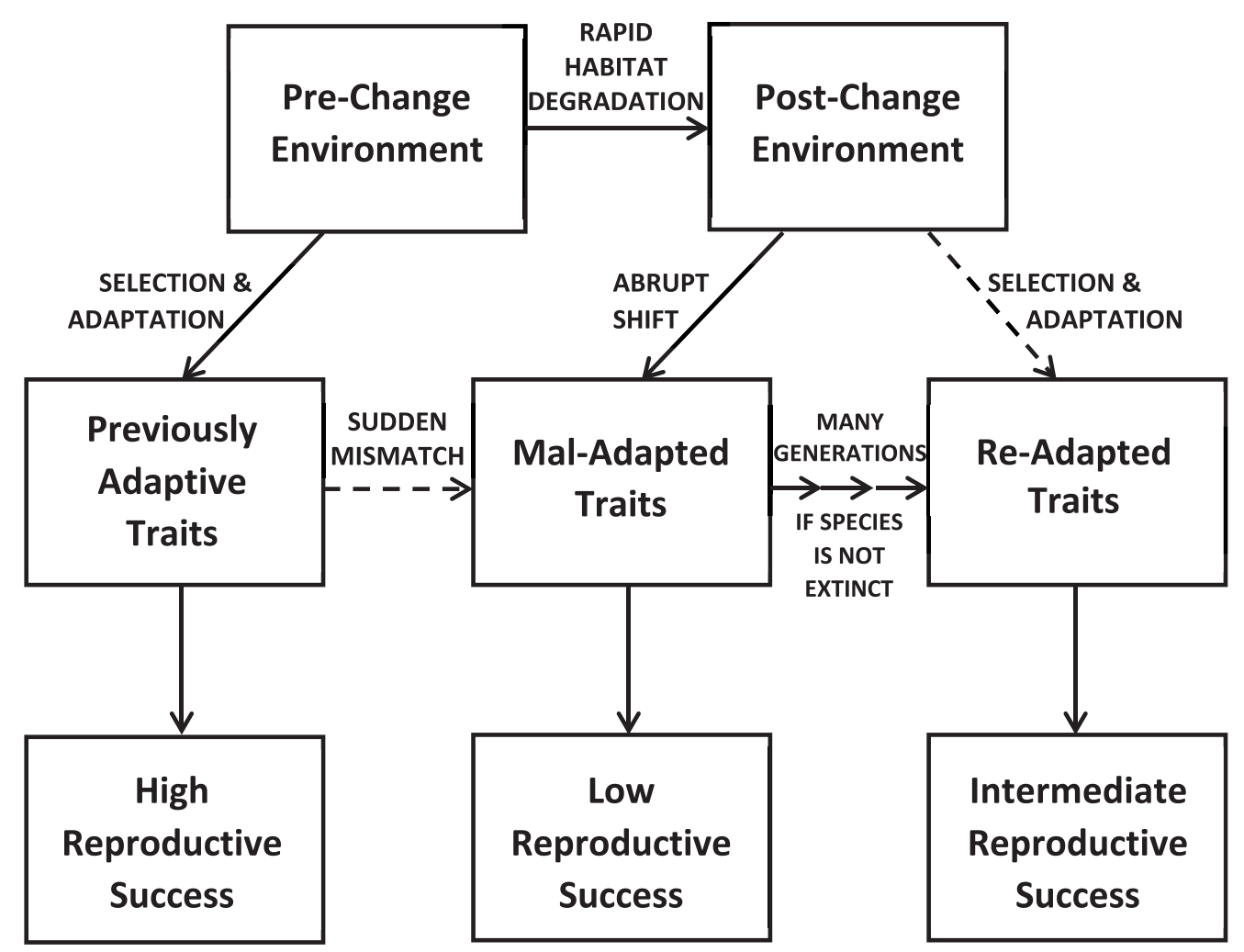

Figure 1: Conceptual diagram of selection and adaptation in response to the rapid habitat degradation modeled in this study. Relationships among environments before and after the habitat change, the fit between traits and environment at three different stages associated with the change (before, immediately after, and many generations after the change, assuming extinction is avoided), and relative reproductive success expected in each. 
lowing adaptation to the new conditions. Specifically, we contrast (1) effects of changes in habitat per se, including reduced habitat availability (i.e., habitat loss) and reduced mean habitat quality (i.e., habitat degradation); (2) increased search costs, including increased mortality risk in the matrix (e.g., associated with habitat alterations that increase mortality risk, or with introduced predators) and increased deferred search costs (e.g., reduced condition due to changes in temperature); and (3) reductions in season duration (e.g., associated with climate change) that can impose limits on time available before settlement. Since environmental changes may differ in their effect on fitness, we investigated what type of change is expected to have particularly large and immediate impact (goal 1). We also asked how these differential impacts depend on species' evolutionary histories (goal 2). Finally, we explored evolutionary readjustment, meaning what affects species' abilities to recover fitness after readaptation (goal 3).

\section{The Model}

\section{Overview}

We imagine dispersers traveling through inhospitable matrix and encountering patches of potentially suitable habitat in a sequence of discrete time steps, while susceptible both to mortality during the search and to deferred costs of searching. Our approach acknowledges that adaptive dispersal and habitat choice involve uncertainty about the availability of different habitats and the costs of movement. Searching among patches is modeled as random and independent among dispersers (i.e., we ignore processes such as the use of social information), and dispersers are able to accurately determine patch quality once patches are encountered.

When a patch is encountered, dispersers choose to settle or to keep searching for a better patch over a season with $n$ time steps. A disperser's choice (based on an optimal settlement threshold) depends on patch abundances and qualities and the costs of dispersal through the matrix. In each step, individuals that survive with probability $s$ either find a patch with probability $h$ or remain in the matrix with probability $(1-h)$, in either case incurring a unit of deferred cost $\delta$ (i.e., a cost imposed after habitat selection, such as reduced mating opportunities or the need to rebuild depleted energy reserves). The results we present in the main text assume that deferred costs increase linearly with time spent searching before settling; we also explore effects of a nonlinear increase in deferred costs in appendix B.

Surviving and settling in a patch results in fitness based on patch quality minus the deferred costs incurred finding that patch. Failure to survive yields zero fitness (i.e., expected number of offspring), $F=0$. Parameters of the model are summarized in table 1, and the code is in author-supplied supplemental material (available online). ${ }^{1}$

\section{The Patch Quality Distribution}

We assume that patch quality (the fitness return value $F$ that would be gained from settling in a particular patch before consideration of deferred costs) is continuous within the interval $\left[0, F_{x}\right]$. Within this interval, patch quality has the frequency density $f(F)$, with each patch encountered having a quality drawn independently from this distribution. Thus, spatial or temporal autocorrelation among encountered patches is ignored.

We use beta distributions to represent patch quality, with a linear remapping from $[0,1]$ to $\left[0, F_{x}\right]$. The shape parameters of the standard beta distribution are set to the same values ( $a=b=4$ ), to provide a symmetric bell curve distribution, as in figure $2 A$. Thus, $f(F)=\beta\left(F / F_{x}\right) / F_{x}$ for $0 \leq F \leq F_{x}$, with $F=0$ otherwise.

\section{Finding Optimal Thresholds Before and After Habitat Change}

Suppose a disperser finds a habitat patch with $F=F_{k}$ in time step $k$. Then the fraction of all patches with fitness higher than $F_{k}$ is

$$
p_{k}=\int_{F_{k}}^{F_{x}} f(F) d F,
$$

and the expected fitness of those better patches is

$$
\varphi_{k}=\int_{F_{k}}^{F_{x}} \frac{F f(F)}{p_{k}} d F .
$$

On visiting a patch at time step $k$, the disperser should settle there if $F_{k}>F_{k}^{*}$, where $F_{k}^{*}$ is the threshold at which the expected fitness from rejecting this patch and continuing to search during the $n-k$ remaining steps exactly equals the fitness to be gained by settling in the patch. The $F_{i}^{*}$ for the steps $i$ during $[1, n]$ constitute a vector of threshold values that can be used either to calculate expected fitness to be gained over all following steps or to simulate the searching process. When habitat change alters one or more of the parameters (see table 1), this previously optimal threshold vector generally results in fitnesses different from those before the change. A new postchange optimal-threshold vector can be found as above with the new parameter values, allowing comparison of dispersal thresholds, results, and fitnesses before the change, after the change (but before adaptation to new conditions), and after readaptation.

1. Code that appears in The American Naturalist is provided as a convenience to readers. It has not necessarily been tested as part of peer review. 
Table 1: Parameters of the model

\begin{tabular}{llcc}
\hline Symbol & \multicolumn{1}{c}{ Definition } & Default magnitude & Range \\
\hline$s$ & Chance of survival over a time step & $.98^{\mathrm{a}}$ & $0-1$ \\
$h$ & Chance of finding a patch in a time step & .5 & $0-1$ \\
$n$ & Number of time steps in a season & $30^{\mathrm{b}}$ & $0-30$ \\
$\delta$ & Deferred cost of dispersing per time step (in fitness units) & $1^{\mathrm{a}}$ & $0-5$ \\
$c$ & Deferred cost exponent & 1 & $.5-2$ \\
$F_{x}$ & Maximum fitness value of a patch (in fitness units) & 100 & $50-150$ \\
$a$ & First shape parameter of a beta distribution & 4 & Fixed \\
$b$ & Second shape parameter of a beta distribution & 4 & Fixed \\
\hline
\end{tabular}

${ }^{a}$ These moderate magnitudes correspond to the intermediate level of patch quality choosiness established in the text. High costs $(s=0.9$ and $\delta=3)$ yield a high level of patch quality choosiness, and low costs $(s=0.999$ and $\delta=0.5)$ result in a low level of patch quality choosiness.

${ }^{\mathrm{b}}$ We might imagine, for example, that natal dispersal involves examining a site for about 1 day (the time step duration), with a total of 30 days available for settlement (season length $n$ ).

' See appendix B, available online.

To find the threshold values of fitness $F_{i}^{*}$ at each step $i$, we need to know the $p_{i+1}^{*}\left(F_{i+1}^{*}\right)$ and $\varphi_{i+1}^{*}\left(F_{i+1}^{*}\right)$. This immediately suggests the need to solve the problem by backward iteration, using the logic of dynamic programming (Bellman 1952). At the final step $n, F_{n}^{*}=0$, because any patch is better than none, and there are no more chances to find a patch. This means that $p_{n}^{*}=p\left(F_{x}\right)=1$ and $\varphi_{n}^{*}=\varphi\left(F_{x}\right)=\bar{\varphi}$, the mean patch fitness.

Now consider step $n-1$. The issue for the disperser is whether the fitness of the current patch $F_{n-1}$ is better than the expectation for the remaining step, which is $\operatorname{sh} p_{n}^{*}\left(\varphi_{n}^{*}-\delta\right)$. So the threshold fitness in step $n-1$ is $F_{n-1}^{*}=\operatorname{sh}_{n}^{*}\left(\varphi_{n}^{*}-\delta\right)$. This allows us to determine $p_{n-1}^{*}=$ $p\left(F_{n-1}^{*}\right)$ and $\varphi_{n-1}^{*}=\varphi\left(F_{n-1}^{*}\right)$ and then the threshold fitness for step $n-2: F_{n-2}^{*}=s h p_{n-1}^{*}\left(\varphi_{n-1}^{*}-\delta\right)+s^{2} h\left(1-h p_{n-1}^{*}\right)$ $\left(\varphi_{n}^{*}-2 \delta\right)$. Here we allow for the possibility of settling either in step $n-1$ or in step $n$. Getting to step $n$ from $n-2$ requires surviving two steps, not settling in step $n-1$, and paying the delayed dispersal cost of both of the last two steps. Proceeding in this way back to step 0 (i.e., just before the first step) yields the overall expected fitness resulting from the entire dispersal sequence, $F_{0}^{*}$, where dispersal decisions at each step from 1 to $n$ are based on the threshold values $F_{1}^{*}$ to $F_{n}^{*}$. In general, in step $k$,

$$
\begin{aligned}
F_{k}^{*}= & s h p_{k+1}^{*}\left(\varphi_{k+1}^{*}-\delta\right)+s^{2} h p_{k+2}^{*}\left(\varphi_{k+2}^{*}-2 \delta\right)\left(1-h p_{k+1}^{*}\right) \\
& +s^{3} h p_{k+3}^{*}\left(\varphi_{k+3}^{*}-3 \delta\right)\left(1-h p_{k+1}^{*}\right)\left(1-h p_{k+2}^{*}\right)+\ldots \\
& +s^{n-k} h p_{n}^{*}\left[\varphi_{n}^{*}-(n-k) \delta\right] \prod_{i=k+1}^{n-1}\left(1-h p_{i}^{*}\right),
\end{aligned}
$$

with the provision that expected fitness at any step in equation (3) cannot fall below 0.

For the results of interest, we generally find a plateau of $F_{i}^{*}$ values for the early time steps and then an abrupt decline to $F_{n}^{*}=0$ as step $n$ is reached (fig. $2 B$ ). This is be- cause the likelihood of reaching the late steps that are influenced by the number of remaining steps can stay very low over all of the early steps (unless survival $\rightarrow 1$ and deferred cost is low), making the threshold magnitude during those steps essentially independent of step number. In these cases, the overall expected fitness $F_{0}^{*}$ and the threshold values on the plateau early in the dispersal sequence are typically the same.

To calculate fitness outcomes and time spent searching for any given scenario, we simulated search and settlement decisions and fitness outcomes for 1,000 individuals, each following the relevant settlement threshold vector for that scenario (see fig. 3 for a few selected scenarios). Mean fitness and variation among individuals in fitness in a given scenario are influenced by several factors. Some individuals die before settling and thus have zero fitness. For individuals that survive to encounter and settle in an above-threshold habitat, their potential fitness (before accounting for deferred costs) can range from the threshold to the maximum $F_{x}$. Their realized fitness, however, is devalued by deferred costs that depend on time to settle. Thus, early in the season, most individuals that survive and settle achieve fitnesses higher than the quality threshold, but because of deferred costs, many individuals that do not encounter suitable habitat until late in the season have fitnesses below the threshold. To address our goals, we ran simulations to contrast outcomes for a broad range of parameter values and quality threshold vectors (see the next section).

\section{Approach to Analysis}

To address goal 1 -whether some types of habitat change have particularly large predicted fitness costs-we compared fitnesses and times spent choosing (or dying in the attempt) before environmental change ("prechange," using optimal-response thresholds) and immediately after habitat 

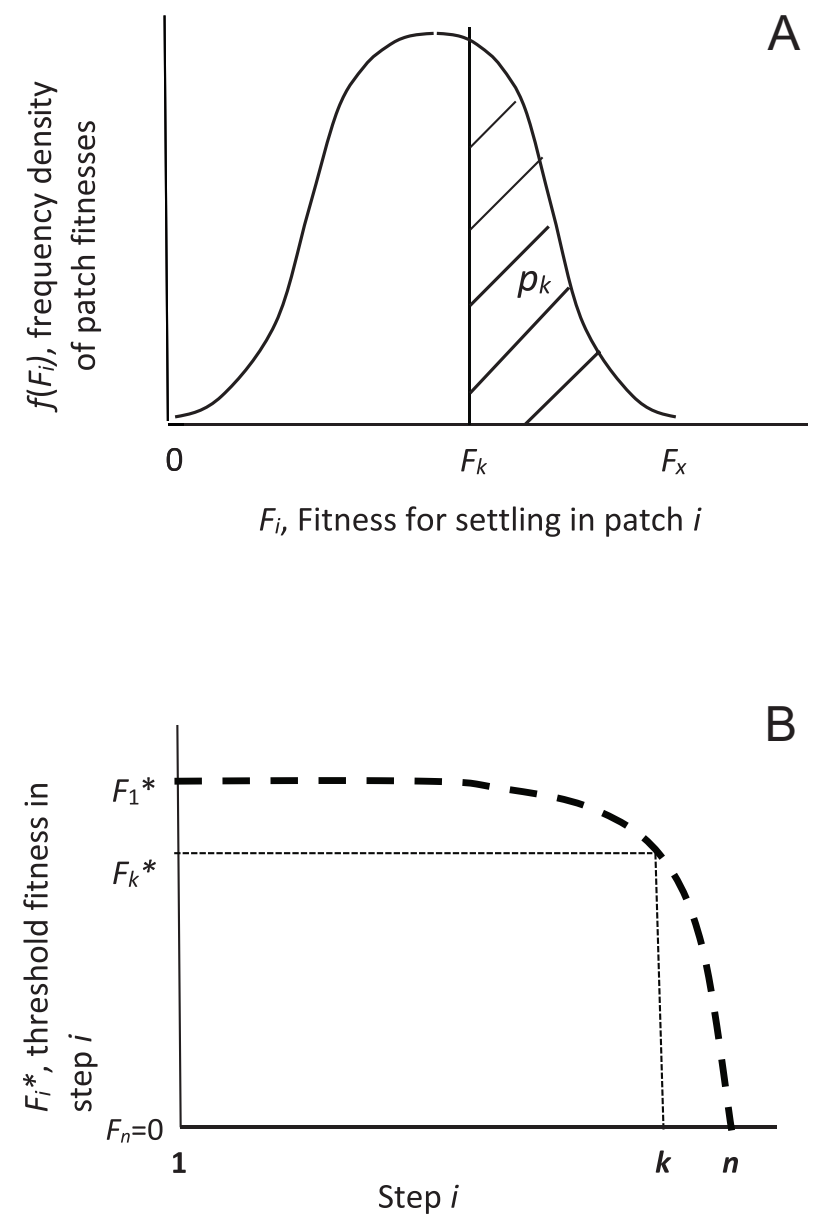

Figure 2: $A$, Frequency density of patch fitnesses (qualities) versus fitness gained by settling in a particular patch. For a habitable patch being found at time step $k$ with quality $F_{k}$, the chance of finding a better patch in each future visit to a different patch is the area $p_{k}$ under the probability density function between $F_{k}$ and $F_{x}$. $B$, Threshold patch quality for settlement $F_{i}^{*}$ at step $i$. This is solved for a vector of optimal threshold patch qualities for each step, starting with $F_{1}^{*}$ and extending to $F_{n}^{*}=0$. Thus, by time step $k$, the optimal threshold for patch acceptance has declined from the initial value $F_{1}^{*}$ to $F_{k}^{*}$.

change ("postchange without readaptation"). We examined scenarios where environmental change manifests itself in one of five ways: (1) a change in the season duration, or number of time steps, $n$, (2) a degradation of average habitat quality modeled as a decrease in $F_{x},(3)$ habitat loss, modeled as a decrease in habitat availability, $h,(4)$ increased deferred costs, $\delta$, per time step during search, or (5) a decrease in survival, $s$, per time step during search. Habitat loss, degradation, and fragmentation often result in reduced fitness via reduced $s, h$, and/or $F_{x}$ and/or increased $\delta$; however, for completeness, we modeled some changes that increase fitness as well. We also examined effects of environmental change that altered the season length (maximum number of time steps, $n$ ), since, for example, a large reduction in season length could be disastrous if animals do not settle quickly enough. We investigated the effects of each of these factors one at a time as well as the effects of a few selected combinations (e.g., where $h$ and $F_{x}$ were simultaneously reduced).

To address goal 2-how evolutionary history might make some species more susceptible to particular types of habitat change - we compared effects of habitat alterations from three baseline (prechange) parameter combinations that resulted in different levels of choosiness about patch quality: $s=0.999$ and $\delta=0.5$ (high choosiness), $s=0.98$ and $\delta=1$ (moderate choosiness), and $s=0.9$ and $\delta=3$ (low choosiness). For each baseline scenario, we determined the optimal patch quality thresholds over all time steps for site selection across a large range of parameter space (goal 1). To address goal 3-the scope for fitness recovery after environmental change-we compared the fitnesses of animals after change without readaptation versus after change with readaptation (i.e., when animals have readjusted their optimal thresholds to match the postchange conditions).

\section{Results}

We first show results from simulations for a few selected scenarios to illustrate habitat settlement dynamics and individual differences in achieved fitnesses across the season (fig. 3). Each panel in figure 3 shows prechange results, and postchange results both before and after readjustment. The three panels address goal 2 by contrasting results for animals that evolved in past conditions that favored high, medium, or low choosiness. Similar simulations were used to address all three goals by calculating mean fitnesses and time to settle (or die) for a broad range of environmental change and response scenarios shown in figures 4-6.

When animals evolved under conditions where search costs were low (high survival, low deferred costs; fig. $3 A$ ), they were very choosy. Their initial habitat quality threshold was 67.3 (relative to the maximum of 100), resulting in only $17 \%$ of habitats encountered being acceptable. The expected number of time steps to settle or die was 9.7; thus, even when quite choosy, animals still almost always settled (or died) well before the late period when they should become less choosy (fig. 3A). When environmental change resulted in a general reduction in habitat quality, before animals adjusted to the new conditions, they were inappropriately choosy: at the beginning of the season, only $0.4 \%$ of habitats encountered were above the threshold. Postchange nonadapted animals mostly rejected habitats until a lowering of the threshold very late in the season rescued some from the trap of maladaptively high choosiness (fig. $3 A$ ). As a result, even though habitat qualities were reduced by only $25 \%$, mean fitness was reduced by about $50 \%$. After adapting to the reduction in 

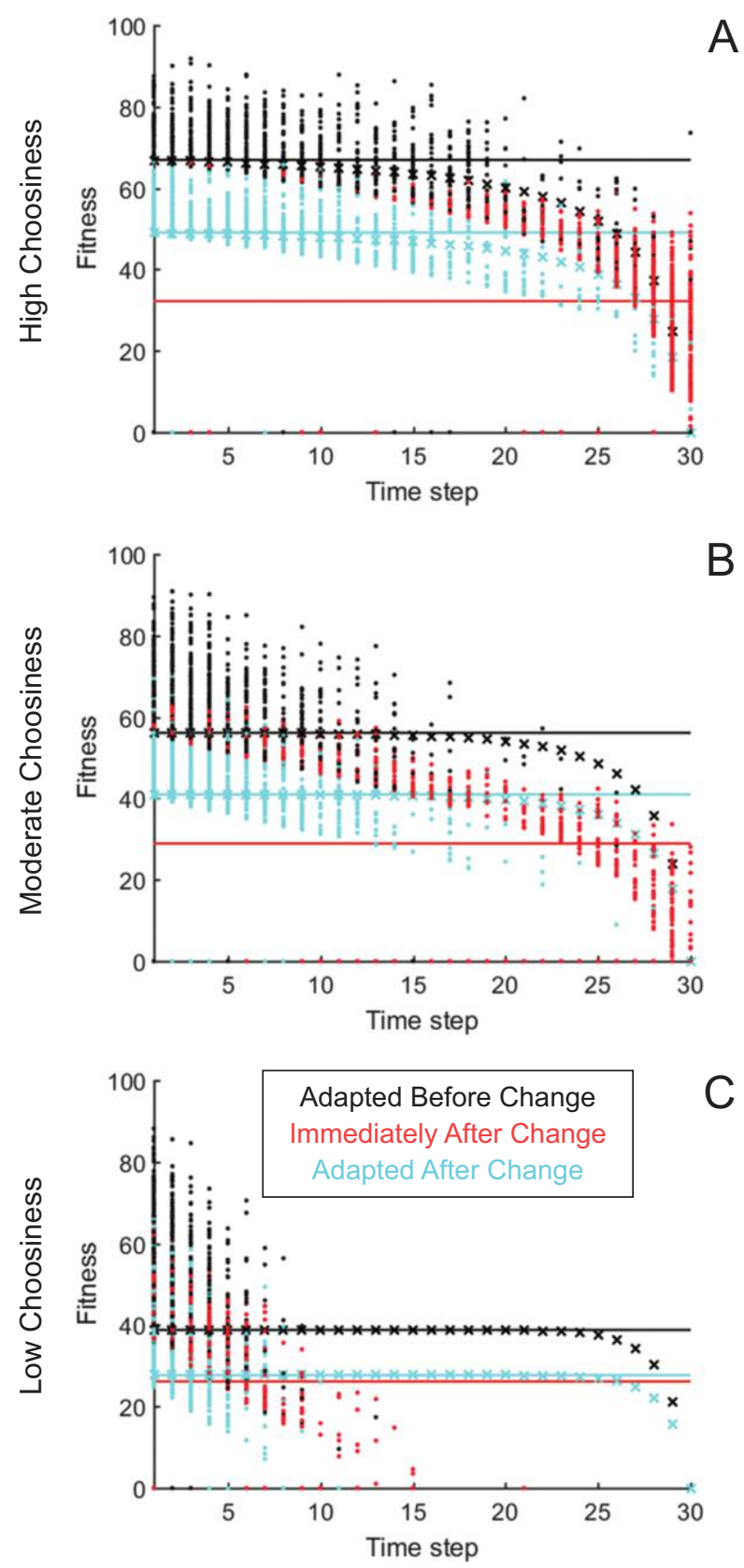

Figure 3: Simulated individual fitnesses (points), and the overall expected fitnesses (horizontal lines) and threshold magnitudes at each time step (marked by a cross of the corresponding color) from equation (3). The simulated change included reduction of the upper limit of patch fitness $\left(F_{m}\right)$ from 100 to 75 . The three panels depict fitness obtained versus the time step at which a patch is chosen or the disperser dies. $A$ is for high choosiness $(s=0.999$ and $\delta=0.5), B$ for moderate choosiness $(s=0.98$ and $\delta=1)$, and $C$ for low choosiness $(s=0.9$ and $\delta=3)$. The points were obtained by simulating the dispersal and patch choice process for 1,000 individuals in each of three scenarios and subtracting the deferred costs for survivors: before change (black), after change but without readaptation (red), and after change and readaptation (cyan). The two adapted conditions (black, cyan) have average habitat quality, animals reduced their quality threshold to 50 , with $19 \%$ of the habitats being acceptable at the beginning of the season and a mean time to settle (or die) of 8.9 time steps. This evolutionary adjustment recovered about half of their lost fitness.

With somewhat higher search costs, animals were less choosy before environmental change (fig. 3B). Even with the reduced choosiness, many of the postchange nonadapted dispersers still rejected patches until the late-season threshold decline in choosiness allowed them to settle. As a result, nonadapted animals suffered a $45 \%$ reduction in mean fitness associated with the $25 \%$ reduction in average habitat quality (only $7 \%$ of patches were acceptable, and the expected number of time steps to settle or die was 13.9). Adaptation to novel environmental conditions again reduced the quality threshold, allowing recovery of about half of the lost fitness (with $40 \%$ of patches acceptable and an expected 4.6 time steps to settle or die).

In contrast, when search costs were much higher, animals were much less choosy before environmental change (fig. $3 \mathrm{C}$ ). Here, only 2.2 time steps, on average, were required to settle or die; that is, most individuals chose a patch (or died) early in the season. Because the pre-habitat change thresholds were fairly low under these conditions, even after environmental change, nonadapted dispersers still often encountered acceptable patches, and most settled relatively quickly ( $46 \%$ of patches were acceptable, and expected time to settle or die was 3.3 time steps). As a result, the $25 \%$ reduction in habitat quality produced only about a $28 \%$ reduction in mean fitness, and thus subsequent adaptation to the new conditions recovered only a very small amount of lost fitness (77\% of patches were acceptable, with 2.3 the expected number of time steps to settle or die). Overall, the same reduction in habitat quality had much less initial negative effect on animals that were adapted to difficult prechange conditions. As a result, those animals required less evolutionary change to adjust to novel environmental conditions.

Note that high costs and low choosiness in the early season are associated with essentially constant thresholds of patch quality until the last few steps (fig. $3 C$ ), because the end of the season is too far in the future to be of great relevance to current decision-making. In contrast, thresholds are moderately influenced by advancing time steps at midseason, when costs are lower and choosiness is higher (fig. $3 A$ ), because late time steps are more accessible and thus of greater influence on decision-making. Intermediate choosiness (fig. $3 B$ ) is more similar to high than to low choosiness.

optimal threshold magnitudes at each time step (crosses), which can be compared with the overall expected fitness for each scenario (horizontal lines). Parameter magnitudes, except as modified within a panel, are $s=0.98, \delta=1, F_{x}=100, h=0.5, n=30$, and $a=b=4$. 


\section{Effects of Altered Habitat Quality and Proportion of Suitable Habitats}

Figures 4 and 5 show effects of two aspects of environmental change that directly involve habitat alteration: changes in habitat quality, $F_{x}$ (fig. 4), and in the proportion of suitable patches, $h$ (fig. 5). When choosiness was high, reductions in $F_{x}$ (to below 100), resulted in sharp drops in the fitness of nonadapted animals (fig. $4 A$ ). The sharp reduction in fitness is due to choosy animals waiting too long before settling (fig. 4B) and often dying before they settle. By adapting to novel environmental conditions (i.e., by reducing their quality threshold), animals adjusted to settle quickly. As a result, their postadaptation reduction in fitness was in proportion to the reduction in habitat quality per se. In contrast, when animals were adapted to difficult conditions before environmental changes, that made them much less choosy: they settled relatively quickly even after environmental change reduced $F_{x}$ (fig. $4 F$ ) and thus suffered proportionally less loss of fitness until $F_{x}$ got very low (so low that even these less choosy animals were still too choosy).

We now turn to the proportion of habitable patches, $h$. For species that exhibit high choosiness before the environmental change, declines in $h$ from its 0.5 benchmark yielded first a modest rate but then an increasing rate of decline in fitness, especially below about 0.2 , (fig. $5 A$ ). This rapid reduction in fitness results from a maladaptive increase in time spent searching (fig. 5B). Again, for animals that adapted to difficult conditions before environmental change by being less choosy, reductions in $h$ caused a relatively small increase in time to settle (or die; fig. $5 F$ ) and thus no dramatic reduction in fitness (fig. $5 E$ ).

A distinction between the two types of habitat shifts (change in habitat quality, $F_{x}$, vs. change in habitat quantity, $h$ ) is the relatively narrow scope for evolutionary adjustment (or perhaps learning) in the $h$ response, as indicated by the modest difference between the fitness curves immediately after environmental change and those after readaptation in figure $5 A, 5 C$. In contrast, the larger gap between the corresponding lines in figure $4 A, 4 C$ suggests greater potential for adaptation (or learning) to mitigate the cost of reduced habitat quality.

\section{Effects of Change-Related Search Costs and Length of Season}

Figure $6 A-6 D$ shows effects of environmental-changeassociated shifts in dispersal-related mortality and the deferred costs that accumulate during dispersal. For prechange comparisons, survival per time step was 0.98 , and the deferred cost per unit search time was standardized to 1 . Not surprisingly, if environmental change makes the matrix more dangerous (i.e., if $s$ is reduced), then fitness declines sharply (fig. 6A). Continuing to use their previously adaptive habitat quality settlement threshold, animals spend less time searching not because they find suitable habitat more quickly but because they have a greater chance of dying before they encounter an acceptable patch (fig. 6B). After adapting to the new, more dangerous matrix conditions, animals are less choosy, settle sooner (fig. $6 B$ ), and are thus able to recover some of their lost fitness (fig. 6A); however, even after they adjust to environmental change, their fitness is still substantially lower than it was before the change.

Increased deferred costs per unit search time also reduced fitness immediately after environmental change; however, because animals tended to settle relatively quickly (fig. 6D) and thus did not have time to accumulate large deferred costs, the negative effect was relatively small (fig. 6C). Adapting to increased deferred costs by being less choosy allowed animals to settle even sooner (fig. 6D), but this only slightly increased fitness (fig. $6 C$ ). In appendix $C$, we also investigate the effect of environmental change altering both the quantity and quality of habitable patches.

The season length within which site selection can be accomplished may also shift as a result of environmental change. It was only when the length of the season was reduced (rather than increased) that fitness implications became important. When animals were only moderately choosy, they generally settled quickly enough that reduced season length had little effect on fitness until the season became very short (fig. $6 E$ ). In contrast, when choosiness was high, even moderate reductions in season length could provide moderately large reductions in fitness (fig. $6 F$ ).

\section{Discussion}

Our model of habitat selection during dispersal combines analytical solutions with simulations to reveal complex interactions between species' evolutionary histories and distinct types of environmental change. We document the fitness impacts of environmental changes and assess the ability of species to readapt to new environmental conditions. Although we also found that environmental change has positive effects on species in some specific contexts, we focus principally on responses to negative environmental changes (e.g., habitat loss or fragmentation, reduced quality of remaining habitat, increased mortality risk while searching for habitat), as we perceive these to be the more important cases. We found, in some cases, that the effects of habitat change were catastrophic, where even optimal postchange behavior would still result in a sharp decline in fitness (figs. 5, $6 A, 6 C)$. But in other cases, habitat selection, together with evolutionary history that shapes choosiness, played a potentially important role in determining the ability to cope with habitat degradation (figs. $4,6 D, 6 E$ ). The novelty of the work 

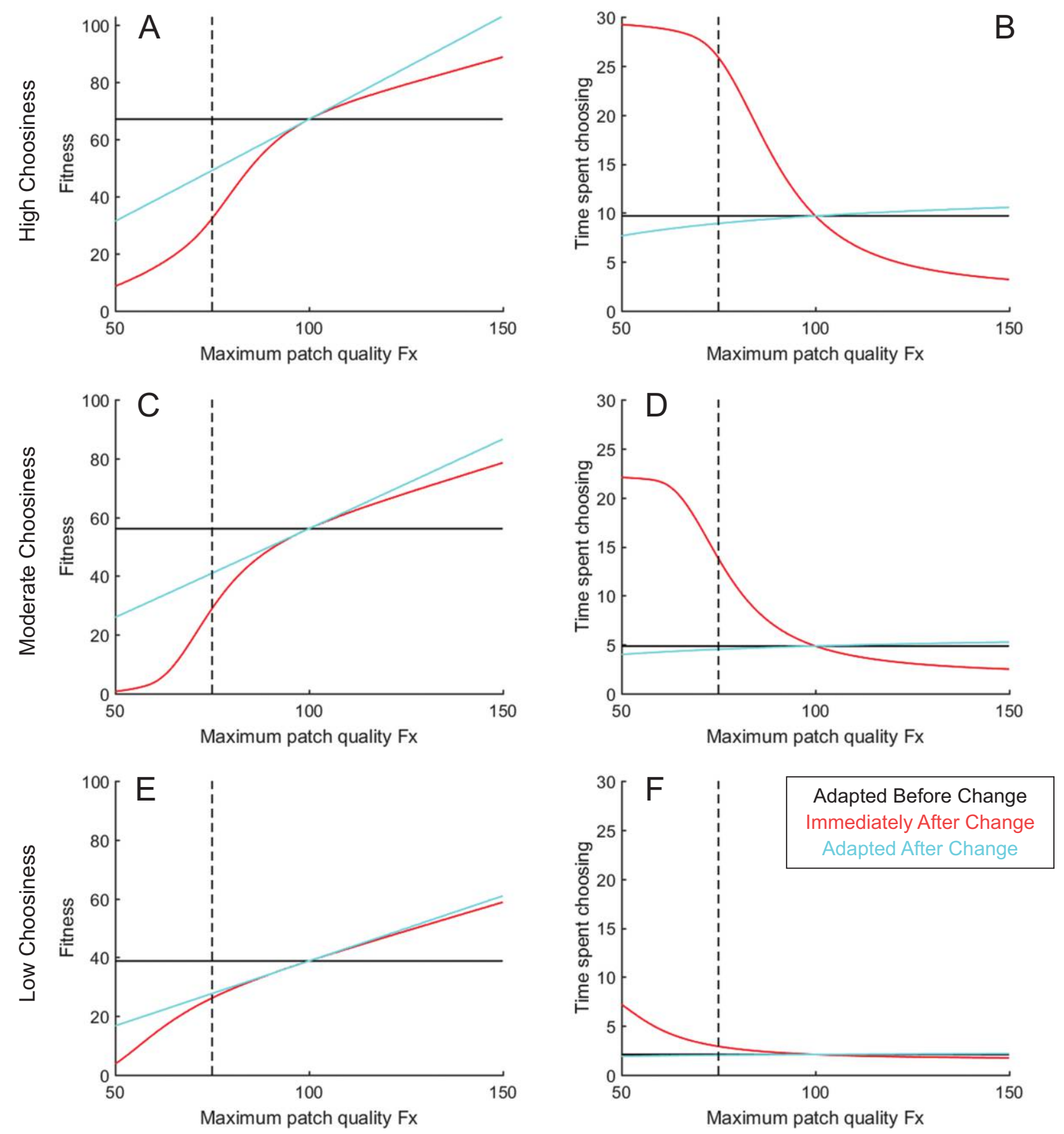

Figure 4: Fitness and time spent choosing for high choosiness $(s=0.999$ and $\delta=0.5 ; A, B)$, moderate choosiness $(s=0.98$ and $\delta=1 ; C$, $D$ ), and low choosiness ( $s=0.9$ and $\delta=3 ; E, F)$ at magnitudes of the maximal patch quality $F_{x}$ from 50 to 150 . The three scenarios in each panel are before change (black), after change but without readaptation (red), and after change and readaptation (cyan). The three lines intersect at the default magnitude where $F_{x}=100$, and the vertical dashed line indicates the magnitude of the shift to $F_{x}=75$. The prechange fitness, based on default parameter magnitudes, provides a benchmark that does not vary along the $X$-axis. Parameter magnitudes, except as modified within a panel, are $s=0.98, \delta=1, F_{x}=100, h=0.5, n=30$, and $a=b=4$. 

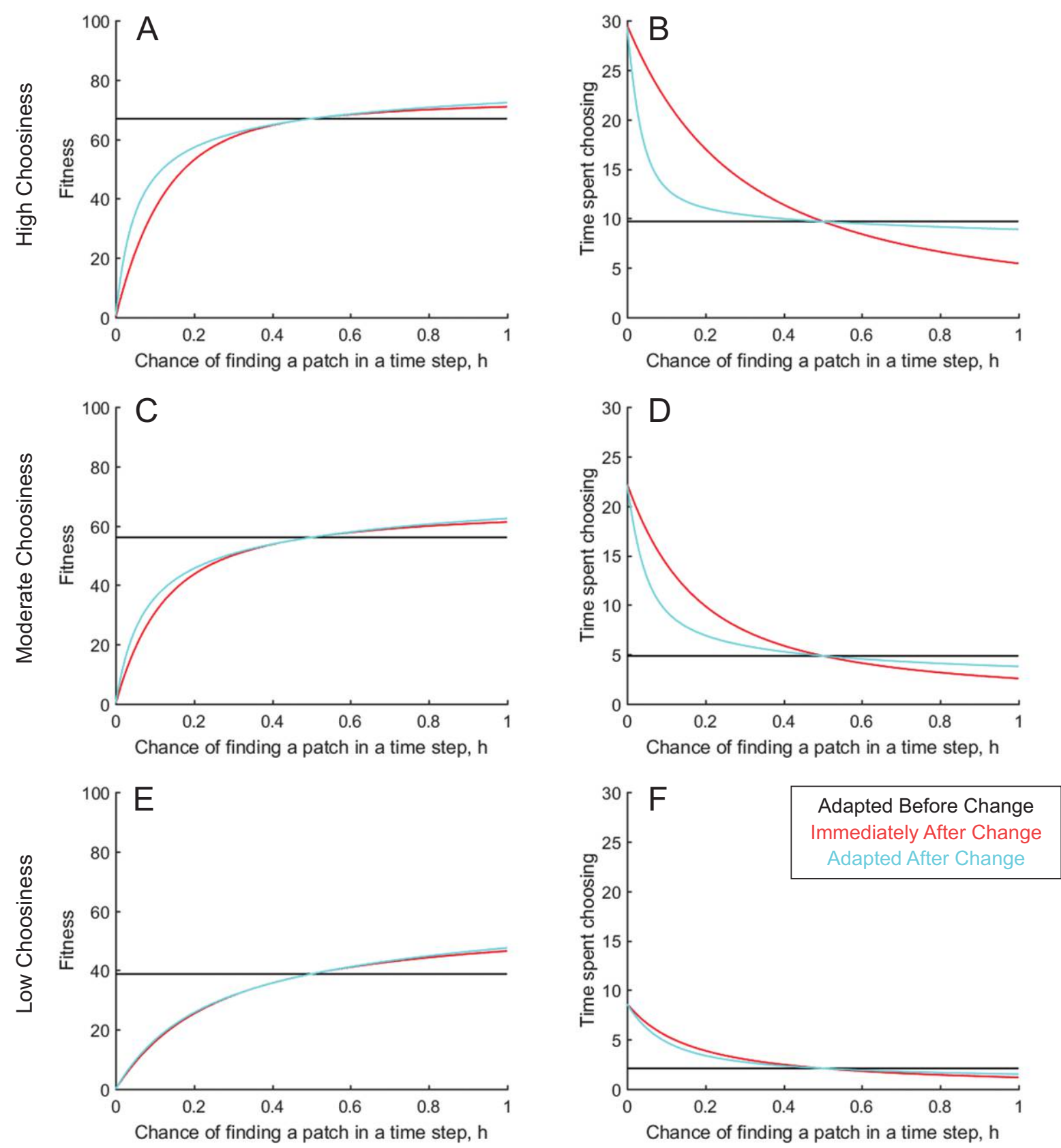

Figure 5: Fitness and time spent choosing for high choosiness $(s=0.999$ and $\delta=0.5 ; A, B)$, moderate choosiness $(s=0.98$ and $\delta=1 ; C$, $D$ ), and low choosiness ( $s=0.9$ and $\delta=3 ; E, F)$ at magnitudes from 0 to 1 of the chance of finding a patch in a time step $h$. The three lines (before change [black], after change without readaptation [red], and after change and readaptation [cyan]) intersect at the default magnitude $(h=0.5)$. The prechange fitness, based on default parameter magnitudes, provides a benchmark that does not vary along the $X$-axis. Parameter magnitudes, except as modified within a panel, are $s=0.98, \delta=1, F_{x}=100, h=0.5, n=30$, and $a=b=4$.

lies in its ability to provide specific predictions on the negative impacts of environmental changes and to demonstrate how these impacts depend on interactions between species traits (i.e., adaptations to their prechange environments) and aspects of environmental change. In addition, the model also identifies situations in which fitness recovery is more likely than in others. Below, we discuss our finding in the context of the three goals framed in the introduction: (1) differential impacts on fitness, (2) the importance of evolutionary history, and (3) the prospects for fitness recovery. 

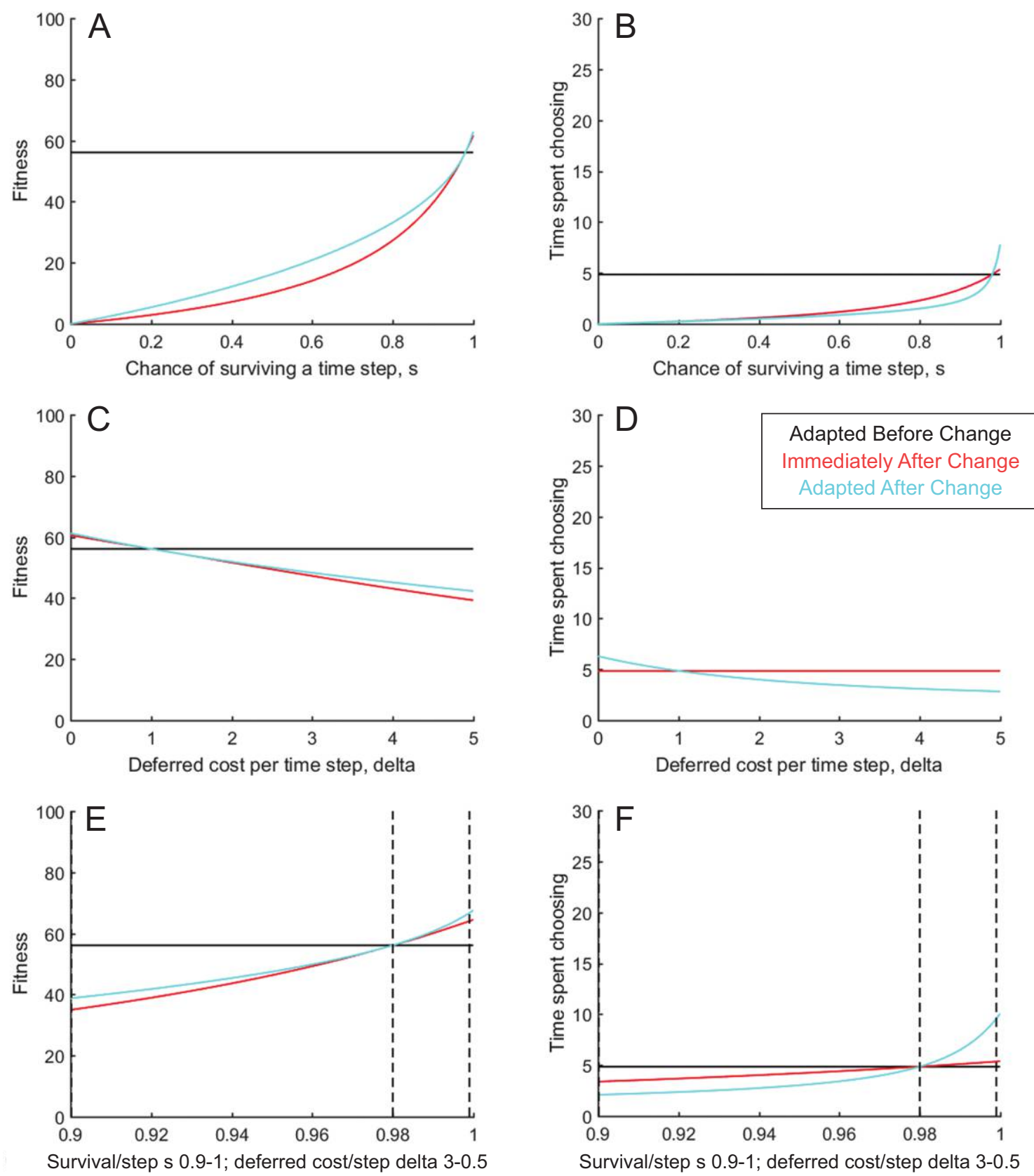

Figure 6: Fitness and time spent choosing for different magnitudes of the chance of surviving a time step $s(A, B)$, the deferred cost per time step $\delta(C, D)$, and the number of time steps in the season, $n(E, F)$. Baseline levels are for a moderate level of choosiness, with parameter magnitudes $s=0.98, \delta=1, F_{x}=100, h=0.5, n=30$, and $a=b=4$. The prechange fitness, based on default parameter magnitudes, provides a benchmark that does not vary along the $X$-axis. As parameters are altered in the panels, the three lines intersect at the baseline levels. In $D$, the black line (of prechange conditions) is masked by the cyan line (of conditions after change and readaptation), as the two are identical. In $E$ and $F$, while survival $s$ varies along the $X$-axis from 0.9 to 1 , deferred cost $\delta$ varies in the opposite direction from 3 to 0.5 ; thus, habitat quality increases by both measures simultaneously from left to right along the axis.

This content downloaded from 128.163.002.206 on August 08, 2020 20:28:56 PM 


\section{Goal 1: Some Aspects of Environmental Change Have Greater Impacts on Fitness than Others}

Our analyses indicate that some types of habitat change reduce fitness more than others - a result not clearly predicted by current theory (though, for some related results, see Fahrig 2007). While the magnitude of reduction differed among species, choosiness levels, and their interaction with specific changes, broadly speaking survival and maximal habitat quality had the strongest negative effects (with a moderate choosiness level and median change levels immediately after change; figs. $4 C, 6 A$ ). When environmental change reduces survival while searching, fitness can be substantially reduced (fig. 6A). An increase of similar relative magnitude in deferred search costs (e.g., by a factor of 5) has an effect on fitness (fig. 6C) similar to that of survival. Of special importance are reductions in habitat quality and quantity (figs. 4,5$)$ that result in large reductions in fitness. In these cases, small to moderate reductions in average habitat quality or quantity (i.e., increased habitat fragmentation or loss) produce only small decreases in fitness; however, the effects accelerate, so further reductions (e.g., when the probability of finding a patch in each time unit drops from 0.5 to 0.2 or lower; fig. 5) result in more severe decreases in fitness. These abrupt fitness declines are due primarily to animals being too choosy and spending much too much time searching before settling. As a result, even with no altered-habitat-related increase in mortality per unit time while searching, many die before settling, with the few survivors often settling only late in the season (figs. $4 B, 4 D, 5 B, 5 D$ ).

\section{Goal 2: Species' Evolutionary Histories Influence Sensitivities to Environmental Change}

Our approach makes predictions on how evolutionary history should affect behavioral responses and fitness costs associated with environmental change. In particular, we predict that overchoosiness that results in large reductions in fitness in recently modified environments should be particularly prominent for animals that evolved in relatively favorable local conditions before change. In contrast, animals that evolved under less favorable conditions before change should be less choosy and should thus generally suffer relatively low fitness costs associated with environmental change. This prediction mirrors the general intuition that species adapted to stressful conditions should be more ready to cope with other sources of stress than those that evolved (or developed) in benign conditions (e.g., Frankenhuis and Del Giudice 2012).

Empirical evidence supports the notion that evolutionary history has shaped habitat settlement behavior. In the environmental-change context, the literatures on ecological traps (Schlaepfer et al. 2002; Robertson et al. 2013;
Hale and Swearer 2016) and on undervalued resources (Gilroy and Sutherland 2007; Patten and Kelly 2010) are relevant. These literatures suggest that evolved, previously adaptive settlement rules cause some animals to use habitats that they should not and others to ignore habitats that they should use. Ecological traps are usually interpreted as arising from misevaluation of habitat quality (e.g., animals settling in poor habitat that they thought was good habitat); however, some cases of maladaptive habitat use revolve around misevaluation of the availability or quality of other habitat sites (Patten and Kelly 2010). This latter type of error more directly fits our model's basic scenario.

Perhaps even more relevant is the literature suggesting that animals that evolved with more suitable habitat or lower risk between patches tend to have higher dispersal tendencies and/or higher choosiness about habitat settlement (Bonte et al. 2006; Fahrig 2007; Van Houtan et al. 2007; Knowlton and Graham 2010). Animals also tend to disperse more or are choosier about settlement sites if they are more mobile or have a larger perceptual range. These animals, in effect, evolved with higher habitat availability and perhaps lower mortality while moving between patches (Knowlton and Graham 2010).

Finally, there is evidence that evolutionary history affects vulnerability to habitat loss in ways that are consistent with our model's predictions. Animals that are less choosy (habitat generalists) are generally less vulnerable to habitat change than habitat specialists (Jiguet et al. 2007; Knowlton and Graham 2010; Crooks et al. 2017). Also, contrary to the idea that more mobile species should be more resilient to habitat loss, when being more mobile is associated with being choosier, more mobile species are more vulnerable to habitat loss (Fahrig 2007; Martin and Fahrig 2016; Tucker et al. 2018). Overall, although studies show that evolutionary history can help explain both patterns of choosiness about habitats and maladaptive habitat settlement after change, no studies that we know of have directly tested our novel predictions on how the combination of evolutionary history and type of change interact to influence the fitness costs of environmental change. Acknowledging these interactions can help explain frequently observed patterns of variation in species response to changes in real-life systems, anticipate future impacts to ongoing changes, and tailor species-specific interventions (Kark et al. 2007; Hale and Swearer 2016).

\section{Goal 3: Prospects for Fitness Recovery after Readaptation Depend on Aspects of Change}

Readaptation to the new, less favorable conditions typically involves becoming less choosy. For some types of habitat change (e.g., reduced maximum and average habitat quality), this adjustment can recover a moderate amount of lost fitness (fig. $4 A, 4 C$ ), but for most types of habitat change (reduced 
habitat quantity, increased search costs), the potential for recovery is low (figs. 5, 6). This difference between types of habitat degradation in their potential for evolutionary recovery is, to our knowledge, a new prediction that can be explained as follows. When rapid habitat change results in a reduction in average and maximum habitat quality (figs. 3,4 ), the loss in fitness immediately after the change is due largely to animals being too choosy and waiting too long for high-quality habitat that is now rare. By simply reducing choosiness, animals can substantially reduce their time spent searching (fig. 4B, 4D). Although animals often end up in somewhat lower-quality habitat when not choosy, this adjustment ensures reasonably high survival. In contrast, when rapid environmental change results in less available habitat, even after adapting to this habitat loss by being less choosy, animals still take a long time to find acceptable habitat and thus still suffer higher mortality before settling. When rapid change involves an increase in mortality rate while searching, the new optimal settlement strategy favors settling almost immediately; however, animals are still unable to recover much lost fitness because they still suffer the higher mortality risk per unit time, and because they are not choosy, they often then settle in relatively poor habitats.

The notion that animals take some time after conditions change before they adjust their choice criteria (i.e., that they initially make suboptimal choices after conditions change) is supported by both empirical and theoretical literature on other choice decisions (e.g., diet choice, patch choice, mate choice). For example, numerous studies show that for some period of time after food availabilities are experimentally altered, foragers that were starved beforehand (i.e., recently experienced low food availability) continue to exhibit different foraging behaviors and diet choices than foragers that were well fed beforehand (e.g., Croy and Hughes 1991; DeMott 1995). Along similar lines, even after predators have left an area, because of a lack of clear information on the change in predation risk, their adaptive decision-making systems mean that prey often remain in hiding (in safe patches) for long periods (Sih 1992).

On the other hand, some studies show that, given time after habitat change, animals have indeed evolved dispersal/habitat selection tendencies that better fit the new overall landscape. For example, classic metapopulation work on butterflies found that increased habitat fragmentation has resulted in a reduced tendency to disperse and reduced search time before settling (Baguette 2003; Schtickzelle et al. 2006). Although these studies document readaptation following environmental change, no studies that we know of have data that test our novel predictions on scenarios that should allow more versus less recovery of lost fitness by readaptation.

\section{Future Directions}

Here, we discuss some ways that our initial, purposely simple model can be usefully extended. One direction for future modeling involves incorporating more details about how animals move through landscapes (e.g., Lima and Zollner 1996; Haddad et al. 2003; Heinz and Strand 2006; Fahrig 2007); how they evaluate predation risk, habitat availability, or habitat quality as they move; and how they actually make their habitat choices (reviewed in Knowlton and Graham 2010). For example (see app. A), Martin and Fahrig (2015) modeled individual movements through a simulated landscape where individuals vary in their path straightness, both in the matrix and within patches, and in their response to patch boundaries. According to the "movement ecology paradigm" (Nathan 2008), other traits that could affect movement and habitat selection include locomotion mode (e.g., flying vs. walking), navigation ability (e.g., perceptual range, the ability to detect patches and even evaluate their quality from a distance), and individual differences in personality (Spiegel et al. 2017). Models incorporating these factors would generate predictions on how individual differences in these traits should influence animals' habitat selection and initial responses to habitat change.

Another future direction involves modeling mechanisms of adjustment after habitat change. Our model focused on the beginning and the end of a potential adjustment process - the behaviors and fitnesses immediately after habitat change (before any adjustment has occurred) and the scope for recovering lost fitness after full adaptation to new habitat conditions. Adjustment could be either via learning or via evolution. Learning could potentially happen almost immediately. Thus, an interesting direction for further model development could examine factors that affect how animals might immediately learn and adjust following rapid habitat change, using, for example, best-of- $n$ or continuous updating approaches (see app. A). It is important to recognize, however, that dispersers may usually have only limited opportunities to learn that the world has changed (Spiegel and Crofoot 2016). If, for example, habitats have always had some poor patches mixed in with good ones, a decision algorithm that updates the animal's settlement threshold on the basis of a few initial encounters with relatively poor patches may lead animals to be too willing to settle in the next poor patch when the overall habitat has not actually changed (i.e., when there is still plenty of good habitat ahead). This potential error would then reduce the tendency for animals to adjust rapidly to actual habitat loss or degradation. This issue may be especially problematic in environments that have remained relatively constant in the past.

Our extant model can also be expanded to explicitly address the evolution of habitat selection thresholds. Our equations allow us to calculate the fitness after habitat change associated with using not just the previously adaptive habitat quality settlement threshold but any level of the settlement threshold. Thus, we can calculate the selection differential for different 
settlement thresholds, which, along with the heritability of the threshold, predicts the evolution of the thresholds. In principle, if we connect our calculated fitnesses to population dynamics, we can examine eco-evolutionary dynamics, including the potential for evolutionary rescue if initial fitnesses immediately after habitat change result in negative population growth (see Holt and Gomulkiewicz 2004).

Another direction for future modeling involves exploration of the effects of combinations of multiple, simultaneous harmful habitat changes. Our analyses focused on the changes one at a time. Future analyses could focus on identifying synergistic negative impacts of simultaneous changes in two or three factors that commonly change in tandem in real systems exposed to human-induced environmental change. For example, habitat degradation might result in both less remaining habitat and higher mortality costs while searching. When might we expect even small changes in these two to combine to produce steep declines in fitness?

Finally, it could be valuable to increase the model's ecological complexity. We could, for example, examine effects of within- and between-season variability on optimal thresholds and fitnesses or density effects on patch quality, with implications for the order of arrival. The latter would make dispersal and settlement potentially both frequency dependent and density dependent (e.g., Schreiber 2012). These complexities could substantially alter both adaptive settlement strategies and predicted impacts of rapid habitat change.

Ultimately, insights from this model and future extensions can facilitate the identification of species at high vulnerability, depending on the mismatch of their evolutionary histories with aspects of contemporary habitat change. Identifying which features of rapid habitat change are more risky can also facilitate efficient use of limited management resources to prioritize the most vulnerable targets over others. Ideally, the models could suggest the types of interventions that would most benefit a particular vulnerable species (e.g., predator playbacks to increase search speed and reduce choosiness vs. predator control to reduce mortality risk while prey move between habitats vs. habitat restoration to increase habitat quality). Of course, much remains to be done both empirically and theoretically to achieve these applied objectives. We hope our initial model provides a useful step along this road.

\section{Acknowledgments}

We thank Dave Westneat and the Crowley and Sih lab groups for comments and suggestions on these ideas and on the manuscript. Two anonymous reviewers, Pim Edelaar, Janneke Hille Ris Lambers, and Judith Bronstein provided well over 100 useful suggestions for revisions. We acknowledge grant support from National Science Foundation Integrative Organismal Systems (1456724; A. S., principal investigator) and the German Research Foundation (DFG) as part of the SFB TRR
(Sonderforschungsbereich/Transregio) 212 ( $\mathrm{NC}^{3}$ [niche choice, niche conformance, niche construction]).

\section{Literature Cited}

Baguette, M. 2003. Long distance dispersal and landscape occupancy in a metapopulation of the cranberry fritillary butterfly. Ecography 26:153-160.

Barnosky, A. D., N. Matzke, S. Tomiya, G. O. U. Wogan, B. Swartz, T. B. Quental, C. Marshall, et al. 2011. Has the Earth's sixth mass extinction already arrived? Nature 471:51-57.

Bellman, R. 1952. On the theory of dynamic programming. Proceedings of the National Academv of Sciences of the USA 38:716-719.

Bonte, D., J. Vanden Borre, L. Lens, and J.-P. Maelfait. 2006. Geographical variation in wolf spider dispersal behaviour is related to landscape structure. Animal Behaviour 72:655-662.

Burns, C. E., and J. S. Grear. 2008. Effects of habitat loss on populations of white-footed mice: testing matrix model predictions with landscapescale perturbation experiments. Landscape Ecology 23:817-831.

Burrows, M. T., D. S. Schoeman, L. B. Buckley, P. Moore, E. S. Poloczanska, K. M. Brander, C. Brown, et al. 2011. The pace of shifting climate in marine and terrestrial ecosystems. Science 334:652-655.

Candolin, U., and B. Wong. 2012. Behavioural responses to a changing world: mechanisms and consequences. Oxford University Press, Oxford.

Crooks, K. R., C. L. Burdett, D. M. Theobald, S. R. B. King, M. Di Marco, C. Rondinini, and L. Boitani. 2017. Quantification of habitat fragmentation reveals extinction risk in terrestrial mammals. Proceedings of the National Academv of Sciences of the USA 114:7635-7640.

Croy, M. I., and R. N. Hughes. 1991. The influence of hunger on feeding behaviour and on the acquisition of learned foraging skills by the fifteen-spined stickleback, Spinachia spinachia L. Animal Behaviour 41:161-170.

DeMott, W. R. 1995. Optimal foraging by a suspension-feeding copepod: responses to short-term and seasonal variation in food resources. Oecologia 103:230-240.

Fahrig, L. 2003. Effects of habitat fragmentation on biodiversity. Annual Review of Ecology, Evolution, and Systematics 34:487-515.

2007. Non-optimal animal movement in human-altered landscapes. Functional Ecology 21:1003-1015.

Frankenhuis, W. E., and M. Del Giudice. 2012. When do adaptive developmental mechanisms yield maladaptive outcomes? Developmental Psychology 48:628-642.

Gilroy, J. J., and W. J. Sutherland. 2007. Beyond ecological traps: perceptual errors and undervalued resources. Trends in Ecology and Evolution 22:351-356.

Haddad, N. M., D. R. Bowne, A. Cunningham, B. J. Danielson, D. J. Levey, S. Sargent, and T. Spira. 2003. Corridor use by diverse taxa. Ecology 84:609-615.

Hale, R., and S. E. Swearer. 2016. Ecological traps: current evidence and future directions. Proceedings of the Roval Societv B 283:20152647. doi:10.1098/rspb.2015.2647.

Heinz, S. K., and E. Strand. 2006. Adaptive patch searching strategies in fragmented landscapes. Evolutionary Ecology 20:113-130.

Holt, R. D., and R. Gomulkiewicz. 2004. Conservation implications of niche conservatism and evolution in heterogeneous environments. Pages 244-264 in R. Ferrière, U. Dieckmann, and D. Couvert, eds. Evolutionary conservation biology. Cambridge University Press, Cambridge. 
Jiguet, F., A.-S. Gadot, R. Julliard, S. E. Newson, and D. Couvert. 2007. Climate envelope, life history traits and the resilience of birds facing global change. Global Change Biology 13:1672-1684

Kark, S., A. Iwaniuk, A. Schalimtzek, and E. Banker. 2007. Living in the city: can anyone become an "urban exploiter"? Journal of Biogeography 34:638-651.

Knowlton, J. L., and C. H. Graham. 2010. Using behavioral landscape ecology to predict species' responses to land-use and climate change. Biological Conservation 143:1342-1354.

Lima, S. L., and P. A. Zollner. 1996. Towards a behavioral ecology of ecological landscapes. Trends in Ecology and Evolution 11:131-135.

Manly, B. F. J., L. L. McDonald, D. L. Thomas, T. L. McDonald, and W. P. Erickson. 2007. Resource selection by animals: statistical design and analysis for field studies. 2nd ed. Kluwer, New York.

Martin, A. E., and L. Fahrig. 2015. Matrix quality and disturbance frequency drive evolution of species behavior at habitat boundaries Ecology and Evolution 5:5792-5800.

- 2016. Reconciling contradictory relationships between mobility and extinction risk in human-altered landscapes. Functional Ecology 30:1558-1567.

Nathan, R. 2008. An emerging movement ecology paradigm. Proceedings of the National Academy of Sciences of the USA 105:19050-19051.

Patten, M. A., and J. F. Kelly. 2010. Habitat selection and the perceptual trap. Ecological Applications 20:2148-2156.

Robertson, B. A., J. S. Rehage, and A. Sih. 2013. Ecological novelty and the emergence of evolutionary traps. Trends in Ecology and Evolution 28:552-560.

Schlaepfer, M. A., M. C. Runge, and P. W. Sherman. 2002. Ecological and evolutionary traps. Trends in Ecology and Evolution 17:474480.

Schreiber, S. J. 2012. The evolution of patch selection in stochastic environments. American Naturalist 180:17-34.

Schtickzelle, N., G. Mennechez, and M. Baguette. 2006. Dispersal depression with habitat fragmentation in the bog fritillary butterfly. Ecology 87:1057-1065.

Sih, A. 1992. Prey uncertainty and the balancing of antipredator and feeding needs. American Naturalist 139:1052-1069.

- 2013. Understanding variation in behavioural responses to human-induced rapid environmental change: a conceptual overview. Animal Behaviour 85:1077-1088.

Sih, A., M. C. O. Ferrari, and D. J. Harris. 2011. Evolution and behavioural responses to human-induced rapid environmental change. Evolutionary Applications 4:367-387.

Sih, A., B. G. Jonsson, and G. Luikart. 2000. Habitat loss: ecological, evolutionary and genetic consequences. Trends in Ecology and Evolution 15:132-134.

Sih, A., P. C. Trimmer, and S. M. Ehlman. 2016. A conceptual framework for understanding behavioral responses to HIREC. Current Opinion in Behavioral Sciences 12:109-114.

Spiegel, O., and M. C. Crofoot. 2016. The feedback between where we go and what we know-information shapes movement, but movement also impacts information acquisition. Current Opinion in Behavioral Sciences 12:90-96.

Spiegel, O., S. T. Leu, C. M. Bull, and A. Sih. 2017. What's your move? movement as a link between personality and spatial dynamics in animal populations. Ecology Letters 20:3-18.

Stamps, J. A., V. V. Krishnan, and M. L. Reid. 2005. Search costs and habitat selection by dispersers. Ecology 86:510-518.

Tucker, M. A., K. Böhning-Gaese, W. F. Fagan, J. M. Fryxell, B. Van Moorter, S. C. Alberts, A. H. Ali, et al. 2018. Moving in the Anthropocene: global reductions in terrestrial mammalian movements. Science 359:466-469.

Van Houtan, K. S., S. L. Pimm, J. M. Halley, R. O. Bierregaard, and T. E. Lovejoy. 2007. Dispersal of Amazonian birds in continuous and fragmented forest. Ecology Letters 10:219-229.

Wong, B. B. M., and U. Candolin. 2015. Behavioral responses to changing environments. Behavioral Ecology 26:665-673.

\section{References Cited Only in the Online Appendixes}

Dombrovsky, Y., and N. Perrin. 1994. On adaptive search and optimal stopping in sequential mate choice. American Naturalist 144:355-361.

Houston, A. I., and J. M. McNamara. 1982. A sequential approach to risk-taking. Animal Behaviour 30:1260-1261.

- 1985. A general theory of central place foraging for singleprey loaders. Theoretical Population Biology 28:233-262.

Janetos, A. C. 1980. Strategies of female mate choice: a theoretical analysis. Behavioral Ecology and Sociobiology 7:107-112.

Janetos, A. C., and B. J. Cole. 1981. Imperfectly optimal animals. Behavioral Ecology and Sociobiology 9:203-209.

Luttbeg, B. 1996. A comparative Bayes tactic for mate assessment and choice. Behavioral Ecology 7:451-460.

2002. Assessing the robustness and optimality of alternative decision rules with varying assumptions. Animal Behaviour 63:805-814.

MacArthur, R. H., and E. R. Pianka. 1966. On optimal use of a patchy environment. American Naturalist 100:603-609.

Mazalov, V., N. Perrin, and Y. Dombrovsky. 1996. Adaptive search and information updating in sequential mate choice. American Naturalist 148:123-137.

Parker, G. A. 1983. Mate quality and mating decisions. Pages 141-164 in P. Bateson, ed. Mate choice. Cambridge University Press, Cambridge.

Real, L. 1990. Search theory and mate choice. I. Models of single-sex discrimination. American Naturalist 136:376-405.

Wittenberger, J. F. 1983. Tactics of mate choice. Pages 435-447 in P. Bateson, ed. Mate choice. Cambridge University Press, Cambridge.

Associate Editor: Janneke Hille Ris Lambers Editor: Judith L. Bronstein 